\title{
Development and validation of a dynamic model for a two-stage speed-reducer
}

\author{
Nicholas J. Tutt ${ }^{1, *}$, Martin P. Venter ${ }^{1, * *}$, and Daniel N.J. Els ${ }^{1, * * *}$ \\ ${ }^{1}$ Stellenbosch University, South Africa
}

\begin{abstract}
This paper presents the development and validation of a six degree of freedom (DOF) dynamic model of a two-stage parallel shaft gearbox, without flaws, which is able to determine the reaction of gearbox components to varying torque inputs and loads. The model utilises flexible shafts and gears rather than using a rigid assumption, to further understand the effect of varying mesh stiffness. The paper replicates the results presented by Diehl and Tang, and improves the number of frequencies that can be analysed. The gear meshing frequencies were expected to dominate the result, however due to the use of a sinusoidal approximation of the varying tooth mesh frequency, the presented model shows the additional gear generated frequencies are present and analysable in the data.
\end{abstract}

\section{Introduction}

Large scale two-stage speed reduction gearboxes are a key component in the operation of air cooled condensers common in minimal water loss systems for utility scale power generation. The varying loads experienced by the fan and gearbox, due to the surrounding structures and the local wind patterns prevalent in this application, are of concern towards the operation and life expectancy of such gearboxes. This paper will develop and validate a six degree of freedom (DOF) dynamic model of a two-stage parallel shaft gearbox, without flaws, which is able to determine how the gearbox's components react to varying torque inputs and loads. The model utilises flexible shafts and gears rather than using a rigid assumption, to further understand the effect of varying mesh stiffness.

Numerical models of multi-stage gear systems have been developed to determine the effect of internal defects to the noise and vibration level of an operational gearbox [1]. These flaws amongst many include: tooth wear, fatigue failure and manufacturing errors [2]. This aids in the the study of gear faults and the development of condition monitoring methods [3].

Widely used standards such as AGMA 2101-D04 [4], empirically develop gear design safety factors to account for all parameters and loads that influence gear life. These safety factors consider dynamic factors that cover the rotational speed and shock loads which although useful, under steady operation conditions. It is found that the full effect of dynamic loads are not completely covered by the standards. The amount of unexpected failures of large scale air cooled condenser fans' gearboxes requires research into the causes of this unexpected

\footnotetext{
*e-mail: nicholas.tutt@gmail.com

**e-mail: mpventer@sun.ac.za

***e-mail: dnjels@sun.ac.za
} 
emergent behaviour. The implementation of the proposed numerical model will provide a tool to do an in-depth analytical analysis into the causes and thus improve the understanding of how torque interacts with a gearbox.

The six DOF model for a two-stage speed reducer, utilising a similar method described in Diehl et al. [5], presented here, is developed. It is then demonstrated that this model can be used as a parametric tool to aid the design of new gearboxes. The research focuses on utilising flexible shafts and gears to give further insight into the dynamic behaviour of the gearbox components.

\section{Gear tooth mesh stiffness}

Gearbox dynamics are characterised by time varying gear mesh stiffness and non-linear backlash [1, 3, 6-9]. The inclusion of such properties ensure that the respective models fully describe the discrete motion of the gears.

Bai et al. [7] review that backlash always exists within gear transmissions due to lubrication, tooth wear, assembly errors, etc. However, backlash is mainly prominent at zero load and load reversals and thus does not form part of this paper's scope.

Time varying mesh stiffness is a main property included in this paper, especially with regards to how it affects the dynamic behaviour of a gearbox [10]. The interaction described between teeth is shown in figure 1, where the forces between the teeth are affected by the effective stiffness that each tooth contributes in reaction to the applied torque load.
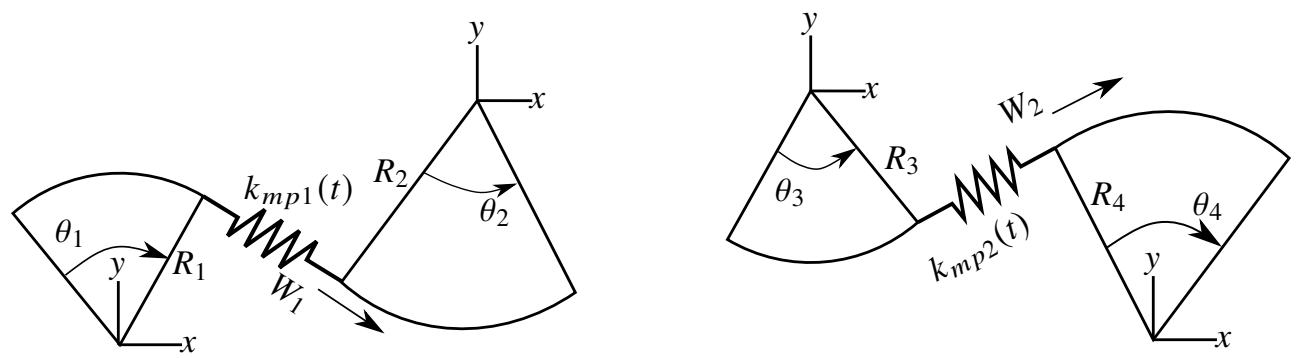

Figure 1. Two-stage meshing freebody diagram showing the forces $W_{1}$ and $W_{2}$

The resistance force, $W_{i}$, generated between the meshing gears is proportionate to the relative displacement of the driving and driven gear in the direction of the meshing line. This is shown in equation (2) with $R$ referring to the gear radius, $\theta$ the relative gear position and $i$ the respective gear stage. The resistance force is given in equation (1); where $k_{m p}(t)$ is the varying mesh stiffness function that cycles according to the mesh frequency $\omega_{i}$, at an amplitude of $k_{v}$ and a mean stiffness of $k_{m}$. The mean mesh stiffness may be determined through experimentation [10], finite element investigation [11], or following standardised procedures such as those outlined in ISO 6336-1:2019 [12].

$$
\begin{aligned}
W_{i}(t) & =k_{m p i}(t) \delta_{i}, \\
\delta_{i} & =R_{p i} \theta_{p i}-R_{g i} \theta_{g i}, \\
k_{m p i}(t) & =-k_{v} \cos \left(2 \pi f_{m_{i}} t\right)+k_{m} \\
i & =1,2
\end{aligned}
$$


The simplified periodic mesh stiffness, $k_{m p}$, characterised by figure 2 is approximated by equation (3). Equation (3) is calculated with respects to the meshing frequency, $f_{m_{i}}$, of the respective gear pairs. The meshing frequencies represent the rate at which tooth pairs mesh and is calculated using equation (4). Where $n_{P_{i}}$ and $n_{G_{i}}$ represents the respective speed of the pinion or gear for the different stages, and $N_{P_{i}}$ and $N_{G_{i}}$ represent the number of teeth of the respective pinion and gear.

$$
f_{m_{i}}=\left\{\begin{array}{l}
N_{P_{i}} \times n_{P_{i}} / 60 \\
N_{G_{i}} \times n_{G_{i}} / 60
\end{array} \quad[\mathrm{~Hz}]\right.
$$

The ideal mesh stiffness profile is currently being experimentally measured. The measurement utilises a set of large module spur gears loaded under quasi-static conditions and recorded using digital image correlation technology. The results will further advise the best method of simulating the mesh stiffness' periodicity.

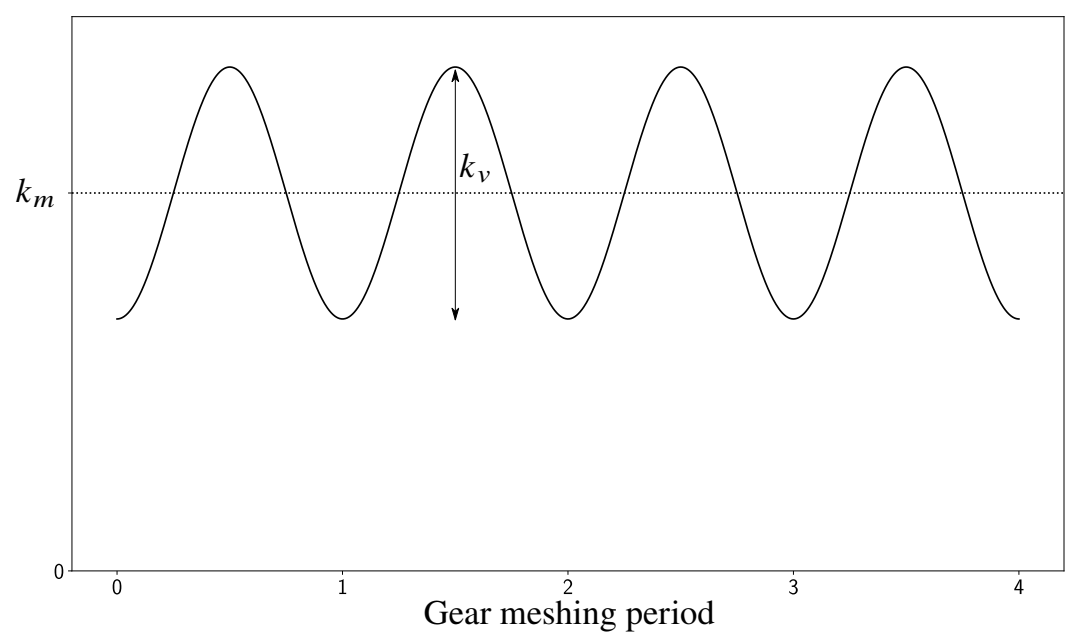

Figure 2. Visualisation of equation (3), which approximates the periodic gear mesh stiffness.

\section{Model development}

A model of the two-stage gearbox is used for concept analysis and validation. Currently gearboxes are designed using standards [13] that are predominantly empirically derived. Although useful for gearboxes, operating at constant speeds with constant loads, the standards fall short in circumstances where there is continuous torque variation coupled with nonuniform shaft bending.

A model which describes the dynamic response of large scale gearboxes under varying dynamic load conditions is needed. A physical gearbox generates the following frequencies [14]: rotational frequency $f_{r}$, meshing frequency $f_{m}$, tooth repeat frequency $f_{t r}$, and assembly phase passage frequency $f_{a p}$. Where the minimum frequency is the tooth repeat frequency 
and the maximum is the mesh frequency. The developed model would need to be able to predict these frequencies under normal operating conditions.

The model presented in Diehl and Tang [5], when analysed, only showcase the meshing frequencies as major contributing factors to the vibration response of the gearbox. The proposed model will aim to ensure that the frequencies outlined in Winterton [14] are present when analysing the gearbox's vibrational response.

The proposed model will utilise the same parameters as those outlined in Diehl and Tang [5], shown in table 1. The module of the gears is specified as $1.59 \mathrm{~mm}$, the speed of the driver is set at $820 \mathrm{rpm}$ and the power of the driver is approximately $2.2 \mathrm{~kW}$.

Table 1. Gearbox model parameters

\begin{tabular}{lcccc}
\hline & Number of teeth & Inertia $\left[\mathrm{kgm}^{2}\right]$ & Damping $[\mathrm{Ns} / \mathrm{rad}]$ & Stiffness $[\mathrm{Nm} / \mathrm{rad}]$ \\
\hline Driver, $I_{D}$ & & $7.51 \mathrm{e}-3$ & & \\
Pinion $1, I_{1}$ & 32 & $8.06 \mathrm{e}-5$ & & \\
Gear $1, I_{2}$ & 80 & $2.97 \mathrm{e}-3$ & & \\
Pinion 2, $I_{3}$ & 48 & $4.00 \mathrm{e}-4$ & & \\
Gear 2, $I_{4}$ & 64 & $1.22 \mathrm{e}-3$ & & $8.20 \mathrm{e} 3$ \\
Load, $I_{L}$ & & $2.73 \mathrm{e}-5$ & & $39.9 \mathrm{ee} 3$ \\
Shaft $1, k_{t 1}, c_{t 1}$ & & & & \\
Shaft $2, k_{t 2}, c_{t 2}$ & & & & \\
Shaft 3, $k_{t 3}, c_{t 3}$ & & & 0.517 & 4.626 \\
Mesh stiffness, $k_{m}$ & & & \\
\hline
\end{tabular}

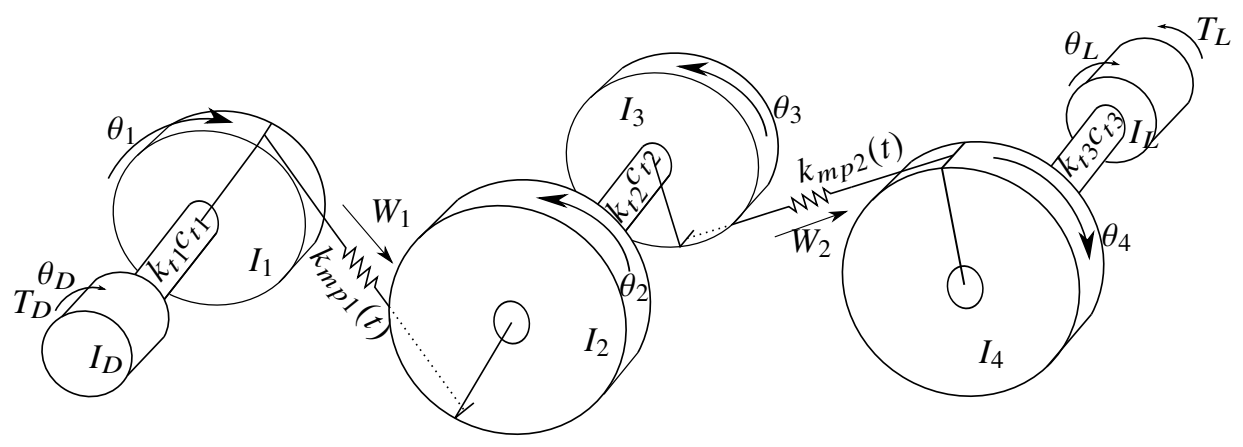

Figure 3. Diagram of the six degree of freedom two stage dynamic gearbox model. 


$$
\begin{aligned}
I_{D} \ddot{\theta}_{D}+c_{t 1}\left(\dot{\theta}_{D}-\dot{\theta}_{1}\right)+k_{t 1}\left(\theta_{D}-\theta_{1}\right) & =T_{D} \\
I_{1} \ddot{\theta}_{1}+c_{t 1}\left(\dot{\theta}_{1}-\dot{\theta}_{D}\right)+k_{t 1}\left(\theta_{1}-\theta_{D}\right) & =-W_{1} R_{1} \\
I_{2} \ddot{\theta}_{2}+c_{t 2}\left(\dot{\theta}_{2}-\dot{\theta}_{3}\right)+k_{t 2}\left(\theta_{2}-\theta_{3}\right) & =W_{1} R_{2} \\
I_{3} \ddot{\theta}_{3}+c_{t 2}\left(\dot{\theta}_{3}-\dot{\theta}_{2}\right)+k_{t 2}\left(\theta_{3}-\theta_{2}\right) & =-W_{2} R_{3} \\
I_{4} \ddot{\theta}_{4}+c_{t 3}\left(\dot{\theta}_{4}-\dot{\theta}_{L}\right)+k_{t 3}\left(\theta_{4}-\theta_{L}\right) & =W_{2} R_{4} \\
I_{L} \ddot{\theta}_{L}+c_{t 3}\left(\dot{\theta}_{L}-\dot{\theta}_{4}\right)+k_{t 3}\left(\theta_{L}-\theta_{4}\right) & =-T_{L}
\end{aligned}
$$

The model, depicted in figure 3, incorporates the dynamics that occur between meshing gears through the inclusion of the meshing forces equation (1). The gear dynamics are assumed to be dominated by the changing mesh stiffness that occur between engaged tooth pairs as discussed in section 2 and further in Chaari et al. [15], Philippe [16] and Pedrero et al. [17].

\section{Model simulation}

Equations (5)-(10) are formulated as a set of matrices (11)-(13). The system of equations are written in Python 3.6, utilising methods from the SciPy version 1.5.2 package [18].

There are six different methods (either implicit or explicit methods) of solving an ordinary differential equation (ODE): the explicit Runge-Kutta methods ('RK45', 'RK23', 'DOP853') are primarily used for non-stiff problems, whereas the implicit methods ('Radau', 'BDF', 'LSODA') are favoured for stiff problems. The stiffness of a problem is not fully defined, however is rather an indication of how fast certain terms within the problem lead to rapid variation of the solution [19].

The specific methods of integration utilised are the explicit Runge-Kutta method of order 5 ('RK45') and the implicit Backwards Differentiation Formula (BDF) which is a multistep variable order ( 1 to 5 ) integration method based on a backward differentiation formula for derivative approximation [20].

$$
\begin{aligned}
& \mathbf{I}=\left[\begin{array}{ccc}
I_{D} & \cdots & 0 \\
\vdots & \ddots & \vdots \\
0 & \cdots & I_{L}
\end{array}\right] \quad \text { (11) } \quad \mathbf{C}=\left[\begin{array}{ccccc}
c_{t 1} & -c_{t 1} & \cdots & \cdots & 0 \\
-c_{t 1} & c_{t 1} & \cdots & \cdots & 0 \\
\vdots & \vdots & \ddots & & \vdots \\
\vdots & \vdots & & c_{t 3} & -c_{t 3} \\
0 & 0 & \cdots & -c_{t 3} & c_{t 3}
\end{array}\right] \\
& \mathbf{K}=\left[\begin{array}{ccccc}
k_{t 1} & -k_{t 1} & \cdots & \cdots & 0 \\
-k_{t 1} & k_{t 1} & \cdots & \cdots & 0 \\
\vdots & \vdots & \ddots & & \vdots \\
\vdots & \vdots & & k_{t 3} & -k_{t 3} \\
0 & 0 & \cdots & -k_{t 3} & k_{t 3}
\end{array}\right](13) \quad \begin{array}{c}
\mathbf{I}+\mathbf{C} \dot{\theta}+\mathbf{K} \theta=\mathbf{T} \\
\theta=\left[\begin{array}{lll}
\theta_{D} & \cdots & \theta_{L}
\end{array}\right]^{T} \\
\mathbf{T}=\left[\begin{array}{lll}
T_{D} & \cdots & T_{L}
\end{array}\right]^{T}
\end{array}
\end{aligned}
$$

Due to the potential stiffness when solving equation (14), the problem was first solved with the RK45 method, followed by the BDF method. The BDF method allows for smaller integration time steps to be taken - preventing rapid variation of the calculated solution. 
The resultant velocity of the last shaft $\dot{\theta}_{L}$ is processed by utilising the Fast Fourier Transform (FFT) algorithm that discretises the velocity signal into its different sinusoidal components and calculates their respective powers. When doing so, it is important to take note of the possibility of alias frequencies in the processed signal, thereby calculating erroneous signal components. Therefore, the inverse of the selected integration time step should be greater than double the maximum frequency expected of the system [21].

As mentioned in section 3, the expected frequencies are the: mesh, rotational, tooth repeat and assembly phase passage frequencies. Due to the meshing forces being a major factor in the modelling of the gearbox, it is expected that the meshing frequencies and their multiples will dominate the generated FFT.

\section{Model results}

The frequency analysis presented in Diehl and Tang [5] show that the meshing frequencies are major contributors to the gearbox model's vibration signal. Their results do not specify whether there are other gear generated spectra displayed in their model, or in their experimental data.

Following the properties outlined in section 3, the model was run for $100 \mathrm{~s}$, with a constant drive and load torque applied. The presented model's FFT results were plotted using the velocity of the last degree of freedom during the model's steady operation. Therefore there were no transient auxiliary frequencies included.

To ensure that minimal alias frequencies were present in the data, the FFT was sampled at $20 \mathrm{kHz}$, with a frequency resolution of $2.4 \mathrm{HZ} /$ bin. Before calculating the FFT, the signal was multiplied by a corrected amplitude Hanning window function [22] to compensate for the amplitude reduction. The Hanning window forces the sample to zero at the start and end of the selected window, thus reducing signal leakage.

The results from the presented model show that on a large scale, figure 4, the meshing frequencies and their multiples are indeed prominent. And when looking at a smaller scale, figure 5, the smaller gear generated spectra are present without being overshadowed.

\section{Conclusion}

The objective of this article is to replicate the six DOF model presented in Diehl and Tang [5] and improve the number of frequencies one could analyse from the model. The developed model shows that nearly all the gear spectra can be read from the processed velocity data generated by the model. When analysing the data on the small scale it was shown that the meshing frequencies do not dominate the calculated frequencies as previously expected, and therefore, the objective of this paper has been met.

Following this article the current model will be further developed to include more translational elements such as those of the bearing locations. By doing so, the effect of the movement caused by meshing can be analysed and the effect thereof can be better understood. 


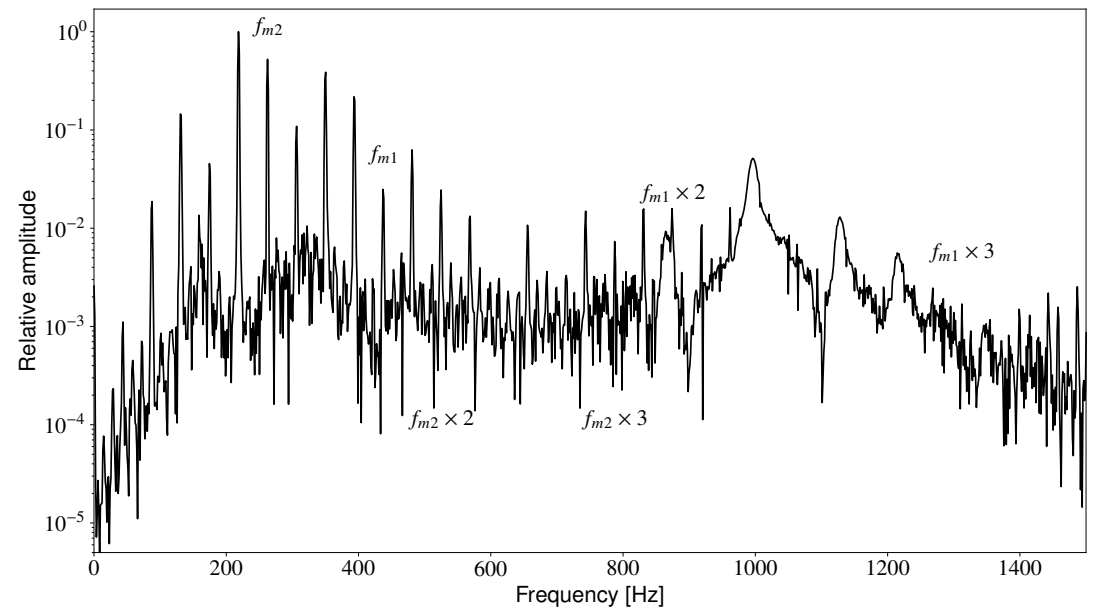

Figure 4. Replicated six degree of freedom model's FFT data (relative to largest amplitude), on a large scale [0 to $1500 \mathrm{~Hz}$ ] showing the meshing frequencies and their multiples. $f_{m 1}$ - first meshing frequency, $f_{m 2}$ - second meshing frequency

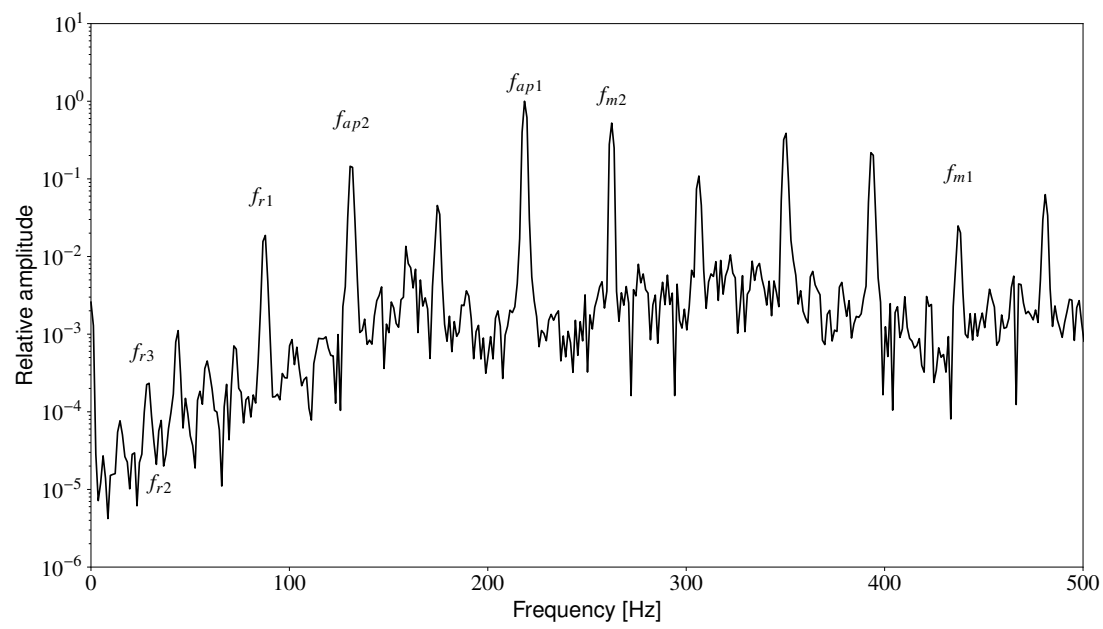

Figure 5. Replicated six degree of freedom model's FFT data (relative to largest amplitude), on a small scale [0 to $500 \mathrm{~Hz}$ ] showing three of the gear generated spectra as specified in Winterton [14]. $f_{m 1}$ - first meshing frequency, $f_{m 2}$ - second meshing frequency, $f_{r 1-3}$ - rotational frequencies, $f_{a p 1-2}$ - assembly passage frequencies

\section{References}

[1] Shengxiang Jia, Ian Howard, and Jiande Wang. The Dynamic Modeling of Multiple Pairs of Spur Gears in Mesh, Including Friction and Geometrical Errors. The International 
Journal of Rotating Machinery, 9(6):437-442, 2003. ISSN 1023-621X. .

[2] Xihui Liang, Ming J. Zuo, and Zhipeng Feng. Dynamic modeling of gearbox faults: A review. Mechanical Systems and Signal Processing, 98:852-876, jan 2018. ISSN 10961216. . URL http://dx.doi.org/10.1016/j.ymssp.2017.05.024.

[3] Farag K. Omar, Kamal A.F. Moustafa, and Samir Emam. Mathematical modeling of gearbox including defects with experimental verification. Journal of Vibration and Control, 18(9):1310-1321, 2012. ISSN 10775463. .

[4] ANSI/AGMA 2001-D04 Fundamental Rating Factors and Calculation Metrhods for Involute Spur and Helical Gear Teeth. Technical report, American Gear Manufactures Association, Alexandria, Virginia, 2004.

[5] Edward J. Diehl and J. Tang. Predictive modeling of a two-stage gearbox towards fault detection. Shock and Vibration, 2016, 2016. ISSN 10709622. .

[6] Grzegorz Litak and Michael I. Friswell. Vibration in gear systems. Chaos, Solitons and Fractals, 16(5):795-800, 2003. ISSN 09600779. .

[7] Zheng Feng Bai, Xin Jiang, Fei Li, and Ji Jun Zhao. Research on Vibration Responses of Gear System with Backlash. pages 55-63. 2020. . URL http://link.springer.com/10. 1007/978-981-15-0238-5\{\\}6.

[8] M. Karray, F. Chaari, M. T. Khabou, and M. Haddar. Dynamic analysis of bevel gear in presence of local damage in nonstationary operating conditions. Lecture Notes in Mechanical Engineering, (207169):325-330, 2018. ISSN 21954364. .

[9] Shengli Zhang, Jiong Tang, and Yu Ding. Modeling and analysis of time-periodic gearbox vibration. Proceedings of the ASME Turbo Expo, 3B:1-8, 2014. .

[10] Naresh K. Raghuwanshi and Anand Parey. Experimental measurement of spur gear mesh stiffness using digital image correlation technique. Measurement: Journal of the International Measurement Confederation, 111(November 2016):93-104, 2017. ISSN 02632241. . URL http://dx.doi.org/10.1016/j.measurement.2017.07.034.

[11] Ph. Sainsot and Ph. Velex. On contact deflection and stiffness in spur and helical gears. Mechanism and Machine Theory, 154, 2020. ISSN 0094114X. .

[12] BS ISO 6336-3:2019 Calculation of load capacity of spur and helical gears. Calculation of tooth bending strength. Technical report, International Organization for Standardization, GENEVA, 2019.

[13] Timothy J. Lisle, Brian A. Shaw, and Robert C. Frazer. External spur gear root bending stress: A comparison of ISO 6336:2006, AGMA 2101-D04, ANSYS finite element analysis and strain gauge techniques. Mechanism and Machine Theory, 111:1-9, 2017. ISSN 0094114. .

[14] John G. Winterton. Component Identification of Gear-Generated Spectra. ORBIT, pages 11-14, 1991.

[15] Fakher Chaari, Radoslaw Zimroz, Walter Bartelmus, Tahar Fakhfakh, and Mohamed Haddar. Model Based Investigation on a Two Stages Gearbox Dynamics under Nonstationary Operations. Condition Monitoring of Machinery in Non-Stationary Operations, pages 133-142, 2012. .

[16] Velex Philippe. On the Modelling of Spur and Helical Gear Dynamic Behaviour. Mechanical Engineering, 2012. ISSN 1448-4846. .

[17] José I. Pedrero, Miguel Pleguezuelos, and Miryam B. Sánchez. Load sharing model for high contact ratio spur gears with long profile modifications. Forschung im Ingenieurwesen/Engineering Research, 83(3):401-408, 2019. ISSN 14340860. .

[18] Eric Jones, Travis Oliphant, Pearu Peterson, and Others. SciPy: Open Source Scientific Tools for Python. URL http://www.scipy.org. 
[19] C. F. Curtiss and J. O. Hirschfelder. Integration of stiff equations. Proc. Natl. Acad. Sci. U S A, 38(1950):235-243, 1952.

[20] Elisabete Alberdi Celaya and Juan José Anza. The integration of stiff systems of ODEs using NDFs and MEBDFs. Journal of Computational Mathematics, 31(2):175-189, jul 2013. ISSN 02549409, 19917139. URL http://www.jstor.org/stable/43693999.

[21] Richard S. Figliola and Donald E. Beasley. 7.2 Sampling Concepts. In Theory and Design for Mechanical Measurements, chapter 7 Sampling, pages 274-275. John Wiley \& Sons, Inc., Hoboken, sixth edition, 2015. ISBN 9781118881279.

[22] T. Irvine. Hanning Window Compensation Factor, 2008. URL http://www.vibrationdata. com/tutorials/Hanning_compensation.pdf. 